\title{
When do psychopathic traits affect cooperative behaviour?
}

\section{An iterated Prisoner's Dilemma experimental study.}

\begin{abstract}
Personality traits have been long recognised to have a strong impact on human decisionmaking. In this study, a sample of 314 participants took part in an online game to investigate the impact of psychopathic traits on cooperative behaviour in an iterated Prisoner's dilemma game. We found that disinhibition decreased the maintenance of cooperation in successive plays, but had no effect on moving towards cooperation after a previous defection or on the overall level of cooperation over rounds. Furthermore, our results underline the crucial importance of a good model selection procedure, showing how a poor choice of statistical model can provide misleading results.
\end{abstract}

\section{Keywords}

Iterated Prisoner's Dilemma game; Cooperation; Psychopathy; Model Selection. Manuscript word count: 5044 


\section{Introduction}

Exogenous factors, such as economic conditions, utilitary outcome and equality have long been considered the principal drivers of human decision-making (Cox, Friedman, \& Gjerstad, 2007; Ohtsuki, Iwasa, \& Nowak, 2015). However, endogenous elements, such as individual propensities, personality traits and emotional conditions have also been recognised to have a strong impact (Boone, De Brabander, \& van Witteloostuijn, 1999; Curry, Chesters, \& Viding, 2011; Loewenstein, 2000). To assess decision-making, several studies adopted some form of economic game, such as the Prisoner's Dilemma (PDG). The PDG is a two-person non-zero-sum game, usually adopted in the non-cooperative form, meaning that players have no opportunity to interact with each other besides playing the game (Scodel, Minas, Ratoosh, \& Lipetz, 1959). In the PDG, two persons each have to decide between two possible actions, giving four possible outcomes (see Table 1). Although the optimal play from a purely economic point of view would be to defect ${ }^{1}$, several studies have recorded cooperative behaviour of the players. Thus, as purely utilitarian considerations did not sufficiently explain players' behaviour (Cox et al., 2007; Declerck, Boone, \& Emonds, 2013), other factors such as reputation (Ohtsuki et al., 2015), punishment and altruism (Cox et al., 2007) have been discovered to influence individuals' behaviour in economic games.

Emotional states and personlity measures have been incorporated into economic models and experiments to reflect the fact that participants in economic games are social human agents (Loewenstein, 2000). Several studies have been carried out regarding the influence of personality on decision-making (Boone et al., 1999). As Ibáñez et al. (2016)(p.1) states: "personality characteristics would play a significant role in different behaviour underlying cooperation". In this work we focus on a specific aspect of human personality, namely

\footnotetext{
${ }^{1}$ In a one-shot PDG, the Nash equilibrium states that the optimal choice is to defect, since no matter what the opponent plays, defection yields a higher payoff than cooperation (Axelrod, 1987).
} 
psychopathy. Psychopaths are described as dominant, superficial, manipulative, affective shallow individuals, unable to form strong emotional bonds with others, and lacking in empathy (Cleckley, 1941). The experimental findings relating such traits to decision-making so far are not entirely clear and sometimes also contradictory. One of the first studies adopting the PDG to assess psychopaths' decisions was Widom (1976): he examined 32 male psychopaths playing 60 trials of the game under two different conditions (communication and non-communication). Unexpectedly, he concluded that psychopaths are capable of cooperating and predicting another person's behaviour. Since then several studies have been conducted to investigate the correlations between psychopathic traits and cooperative behaviours. Criminal psychopaths from a high security psychiatric hospital displayed significantly higher proneness to competitive behaviour in an iterated PDG (Mokros et al., 2008), choosing selfish instead of cooperative strategies more often that healthy adults. Furthermore, a negative correlation between cooperation and psychopathic traits was found in male (but not female) participants in a general population sample (Rilling et al., 2007). Results suggested that some aspects of psychopathic traits were specifically predictive: primary psychopathy (low-anxious, usually viewed as a direct consequence of some intrinsic deficit), Rebellious Nonconformity (e.g. the propensity to do something others might judge as inappropriate) and Machiavellian Egocentricity (e.g. the willingness to trick someone for one's own sake). Furthermore, results have suggested that different aspects of psychopathy affect cooperation differently, when looking at PDG and bargaining games (Curry et al., 2011). Primary, but not secondary, psychopathy was found to predict lower acceptance rate of unfair ultimatum game offers, and lower offers in a dictator game in a sample of 47 prisoners (Koenigs, Kruepke, \& Newman, 2010). Moreover, psychopathic, high disinhibited and impulsive personality traits are predictive of a lack of reciprocity in a trust game (Ibáñez et al., 2016). These dissociative effects of psychopathic traits could be reflected better in a model 
that directly differentiates disinhibition, meanness (lack of care about other individuals) and boldness (risk-seeking and fearlessness), as the Triarchic Model of Psychopathy does (C. Patrick, Fowles, \& Krueger, 2009).

According to these results, people scoring high in psychopathic measures (primary and/or secondary) are expected to adopt less generous and more selfish behaviours, to aim to maximise their own profit, and not to care about the opponent compared to healthy individuals,. In this study, we use an iterated version of the game (IPD) to investigate the effect of personality traits emerging over time. We chose to adopt an iterated version rather than a oneshot PDG because: (1) the effect of personality becomes more evident in repeated games (Boone et al., 1999) and individuals cannot be fully analysed in one-shot games (Axelrod, 1987); (2) we investigate how cooperative behaviour changes from one trial to the next. In the current study, facial expressions of the opponents are presented to the player after each round. The purpose is to create an environment in which players have some sort of connection with their opponent, as opponent characteristics were discovered to have a strong effect on a player's behaviour. However, there is no manipulation of those facial expressions, and we are not testing the effect of those feedbacks on players' decisions. Thus, with this experiment we aim to capture the effect of psychopathic traits on decision-making processes in the presence of facial feedback. Our hypothesis is that players showing lower levels of psychopathy will adopt more cooperative behaviours, compared to players scoring high in the psychopathic measures. 


\section{Methods}

\subsection{Sample}

A total of 398 participants were recruited through social media, email lists and adverts on a University campus.

The experiment was conducted on an online platform, and complete data were available for 378 participants. Participants gave informed consent for participating in an online game. They received a small compensation based on their achieved sum from the game, with each point they accumulated being awarded $€ 0.10$ by means of an Amazon voucher. Participants who took too short or too long time (Mean $\pm 3 * \mathrm{SD}$ ) to complete the task were excluded from the study, leading to the exclusion of 4 participants. Moreover, 60 participants did not compile the personality questionnaire in a consistent way and were excluded by the analysis. The final sample was composed of 314 participants ( 180 females, mean age $=27.41$ ). See Table 3 for sample descriptive statistics.

Informed consent was obtained from all participants prior to starting the game and participants were debriefed after the game regarding the opponent being PC-based rather than a real other participant. We obtained ethical approval for the study from the German Psychology Association ethics board.

\subsection{Material}

\subsubsection{Experiment procedure}

After having filled in a short questionnaire on socio-demographic data, the participants were informed about the structure of the game. Each participant played a 15 trial Iterated Prisoner's Dilemma game. The game was presented as a commercial game in which players were the owner of a shop situated next to another store, selling various technical goods. Al- 
though participants played against a computer throughout the whole game, they were told they were playing against a human opponent. The opponent was represented by a video clip of a person, taken from the Denver Intensity and Spontaneous Facial Action (DISFA) Database (Mavadati, Mahoor, Bartlett, Trinh, \& Cohn, 2013). The opponent was introduced at the beginning of the game showing a 300ms video of a person with a neutral expression, after showing a "wait until we connect you to another player" instruction. In each trial, the participant was asked to decide whether she wanted to sell the good at the standard price (cooperation) or at a sale price (defection). They were encouraged to maximise their own profit, in order to achieve the most points at the end of the game. Four different outcomes were possible, according to the players' decisions: percentages in Table 2 show the profit and 4,3,2 or 1 points were awarded according to the profit earned in each trial.

The computer was programmed to play a tit-for-two-tats strategy, meaning that it cooperates until the opponent defects twice in a row, then it defects until the player cooperates again. In the last two rounds however, the computer was programmed to defect, as an attempt to simulate human behaviour. A page with the two decisions made and the consequent payoff was shown after each round. In addition to the payoff, participants were shown a short video of facial feedback, supposedly from the webcam of the opponent. Videos were taken from the Denver Intensity and Spontaneous Facial Action (DISFA) Database (Mavadati et al., 2013). Two individuals were selected (one female and one male) to represent the opponents and 10 different $300 \mathrm{~ms}$ snips were created for each of the female and the male persons and for each of the happy and sad expressions. For each cooperation, the participant was presented with a happy facial expression video of their opponent, while they were presented with a sad facial expression video of their opponent after each defection. 


\subsubsection{Questionnaires}

At the end of the game, a questionnaire was presented to the participants asking whether they believed in the setting of the game and which strategy they adopted. Participants were asked to state how much they believed they were playing against a real person, choosing a state in a scale from 1 to 5 ( 1 not at all, 5 completely). Participants were also asked whether they were trying, on a scale from 1 to 5, to maximise their own profit. Lastly, they were asked to fill in the Triarchic Model of Psychopathy questionnaire (TriPM) (C. J. Patrick, 2010).

In order to control for potential confounders related to anxiety, aggression and depression, we included the Buss Perry Aggression Questionnaire (Buss \& Perry, 1992), the Penn State Worry Questionnaire (van Rijsoort, Emmelkamp, \& Vervaeke, 1999), the Mood and Anxiety Symptom Questionnaire (Casillas \& Clark, 2000) as well as the Brief Depression Severity Questionnaire (Kroenke, Spitzer, \& Williams, 2001). Measures of the PDG were not related to any of these variables, therefore we did not include them in further analyses.

\subsubsection{Psychopathy measures}

Psychopathy was assessed through the Triarchic Model of Psychopathy questionnaire (TriPM). A reliability test (Cronbach's alpha) in the current sample shows acceptable internal consistencies (Table 3). Developed by Patrick, Fowles, \& Krueger (2009), the questionnaire is based on three factors: disinhibition, boldness, and meanness.

Disinhibition describes a general propensity toward impulse control problems, involving a lack of self-control, weak restraint and difficulties in regulating emotion (Patrick, Drislane, \& Strickland, 2012; Patrick \& Drislane, 2015). High level of disinhibition-related behaviour includes irresponsibility, impatience, impulsive actions leading to negative consequences, alienation and distrust, untrustworthiness, proneness to drug and alcohol problems, and en- 
gagement in illicit or other norm-violating activities.

Boldness suggests a capacity to remain calm and focus in situations involving pressure and threat. It can be associated with social dominance, low stress reactivity and thrill-adventure seeking (Benning, Patrick, Hicks, Blonigen, \& Krueger, 2003), and it can be recognised in manifestations of imperturbability, assertiveness, persuasiveness, and social poise.

Meanness entails deficient empathy, lack of close social attachment towards others, rebelliousness and empowerment through cruelty. Individuals with high meanness scores usually show callousness, cold-heartedness and apathy towards others (Patrick, Drislane, \& Strickland, 2012). The notion of meanness is central to conceptions of psychopathy in criminal and delinquent samples (Patrick, Fowles, \& Krueger, 2009).

\subsection{Statistical Analysis}

The variables under investigation were the total percentage of cooperation, the percentage of cooperation after a previous cooperation $(\mathrm{CaC})$, and after a previous defection $(\mathrm{CaD})$. The total percentage of cooperation was calculated as the mean percentage of cooperative decisions over the 15 trials; while $\mathrm{CaC}(\mathrm{CaD})$ was the percentage of times a participant cooperated immediately following a previous cooperation (defection). The same analyses were implemented on the three dependent variables in parallel. The explained variables were regressed against a fixed set of explanatory variables which included: gender ( $1=\mathrm{fe}-$ male,2=male) as it has been found to be correlated with psychopathic traits (Deutsch, 1990; Hess, Blairy, \& Kleck, 2000); age to control for age effects; the believe variable which accounted for the participants' level, on a scale from 1 to 5 ( $1=$ not at all, $5=$ completely), of belief that they were playing against a fellow human; and the maximise variable that expresses the extent to which participants were trying to maximise their own profit during the game ( $1=$ not at all, $5=$ completely). The three dimensions of psychopathic personality, meanness, 
boldness and disinhibition were included as dimensional scores. The mean and variance of these measures in our sample are similar to other samples previously collected (Almeida et al., 2015). Summary statistics for the predictor variables and a correlation matrix amongst dependent and independent variables are provided in Tables 3 and 4.

The analysis of the data can be divided into three phases. First, candidate models were fit to half of the data in order to identify suitable models for the dataset. Since all three DVs were produced from a series of zeros and ones, we considered a Generalised Linear Model (GLM), a Logistic (LM) and a Beta-Binomial (BBM) regression models as candidate and compared their fits to data. Secondly, repeated k-fold cross-validation was implemented to select the best predictive models from among these candidates. Finally, the selected model was fitted on the complete dataset. All the analyses were implemented in R. Full details of the statistical analysis are given in supplementary material.

\section{Results}

We first analysed the total rate of cooperation over the fifteen rounds as dependent variable (Table 5). The only variable with a statistically significant impact at the 5\% level on predicting the overall cooperation is maximise. Participants who aimed to get the highest score possible for themselves were less cooperative than people who were not focused on their gain. In this sense, their behaviour could be considered rational although it has been proven that cooperative behaviour leads to higher rewards than a negative strategy in an iterated game (Mao, Dworkin, Suri, \& Watts, 2017). Personality traits are not a significant predictor (at the 5\% level) when looking at the overall cooperation.

Disinhibition is the only significant personality aspect when considering the maintainance of cooperative behaviours between trials $(\mathrm{CaC})$ (Table 5). The less inhibited players were, the more they switched strategy from cooperation to defection. In this sense, more disinhibited 
players tend to be less consistent in cooperative strategies.

There is no effect of personality traits on the rate of cooperation after a previous defection (Table 5), though belief increases cooperation. Players tend to switch from defection to cooperation more often when they believed in the experimental set up, thinking they were playing against another fellow human being.

Furthermore, through the analysis of the dataset we observed the importance of selecting the model with the best fit to the data. The necessity of such a procedure is highlighted by the fact that results from the poorly fit binomial regression would have been very misleading for our dataset: it shows several predictors as being statistically significant and with greater significance levels than for the best fit models (see Appendix A).

\section{Discussion}

Our results seem to partially contradict our initial hypothesis that cooperation might be dependent on the player's psychopathic traits. The only impact of psychopathic traits on player's behaviour was found with regard to the maintenance of cooperative behaviour, but not on the overall tendency of players to cooperate, which is inconsistent with previous findings using a similar setup (Rilling et al., 2007; Curry et al., 2011).

In our study, more disinhibited individuals engaged a less continuously cooperative strategy, switching to defection more often than participants with less disinhibited players. As disinhibition is conceptualized as irresponsible, impatient and impulsive tendencies, related to antisocial behaviour, these results match previous findings that point to a relationship between rebellious nonconformity and defection (Mokros et al., 2008). Furthermore, a recent study found a positive association between disinhibition and risk-taking in a loss context (Maes, Woyke, \& Brazil, 2018). Contrary to our expectations, meanness was not a significant predictor in our models, indicating a lower behavioral relevance of that aspect as pre- 
viously found (Balash \& Falkenbach, 2018). On the other hand, personality traits do not show any effect on the decision to switch to cooperation after a previous defection or on the overall cooperation.

A possible explanation for the lack of an effect of psychopathic traits on cooperation in our experiment is that the majority of the participants (165 out of 314) strongly believed they were playing against a computer (scoring 1 out of 5 for belief) and only 11 participants completely believed they were playing against a real person (scoring 5 out of 5 for belief). Personality traits may not play as much of a role when playing against a computer as they do when interacting with a person. This problem is due to the limitation of the study using an online data collection approach. Thus, design improvements will be necessary to create an environment in which participants can have a more realistic interaction with the opponent. This will make it possible for the participants to totally engage in the game and higher variations could be observed between high and low psychopathic subjects. Moreover, it would be useful to control for other personality dimensions in future works, such as the big five personality traits.

An interesting outcome of our experiment is the correlation between the willingness to maximise the outcome and the strategy adopted: participants defected more overall if they were trying to maximise their own paayoff, compared to less profit-oriented players. However, as we measured the motivation to maximise profit only with one item in the questionnaire, this result needs further investigation with more detailed evaluation of the strategic goals of individuals in the game.

Furthermore, we want to emphasise the importance of an appropriate model selection technique. In appendix A we report a very interesting finding: fitting a binomial model would have suggested that several predictors were indeed statistically significant for overall cooperation and with greater significance levels than we observed with our best fit models. Since 
the binomial model was initially considered the most appropriate regression, but revealed through repeated k-fold cross-validation to be a poor fit to data, this highlights the importance of a rigorous model selection procedure to avoid overstating outcomes and provide a reliable interpretation of experimental results.

Our findings show not only the crucial impact of model selection in the analyses, but also the impact of general game behaviour and belief on the outcome in a game theory paradigm of cooperation. Future research therefore should not only investigate specific game behaviour but also take into account participants' belief about the experiment (Kätsyri, Hari, Ravaja, \& Nummenmaa, 2013). Other confounding variables in such a task could be cognitive and motivational factors, which we were not able to control for. Future investigations should include pre- and post-measurement of such factors, e.g. related to state stress. Our study adds to the discussion on the use of deception in economic game research (Bonetti, 1998) and highlights the relevance of further investigation of such effects. Despite these limitations our study shows evidence for a relationship between psychopathic personality traits, specifically disinhibition, with a switch to defection instead of towards cooperation, but no overall relevance of psychopathic traits for cooperation behavior in the game. Furthermore, the change of results based on statistical model selection show that findings from such experimental designs including dimensional scores of personality measures can be instable and models should be carefully selected. 


\section{References}

Almeida, P. R., Seixas, M. J., Ferreira-Santos, F., Vieira, J. B., Paiva, T. O., Moreira, P. S., \& Costa, P. (2015). Empathic, moral and antisocial outcomes associated with distinct components of psychopathy in healthy individuals: a triarchic model approach. Personality and Individual Differences, 85, 205-211.

Axelrod, R. (1987). The evolution of strategies in the iterated prisoner's dilemma. The dynamics of norms, 1-16.

Balash, J., \& Falkenbach, D. M. (2018). The ends justify the meanness: An investigation of psychopathic traits and utilitarian moral endorsement. Personality and Individual Differences, 127, 127-132.

Benning, S. D., Patrick, C. J., Hicks, B. M., Blonigen, D. M., \& Krueger, R. F. (2003). Factor structure of the psychopathic personality inventory: validity and implications for clinical assessment. Psychological assessment, 15, 340.

Bonetti, S. (1998). Experimental economics and deception. Journal of Economic Psychology, 19, 377-395.

Boone, C., De Brabander, B., \& van Witteloostuijn, A. (1999). The impact of personality on behavior in five prisoner's dilemma games. Journal of Economic Psychology, 20, 343-377.

Buss, A. H., \& Perry, M. (1992). The aggression questionnaire. Journal of personality and social psychology, 63(3), 452.

Casillas, A., \& Clark, L. (2000). The mini mood and anxiety symptom questionnaire (mini-masq). In Poster presented at the 72nd annual meeting of the midwestern psychological association, chicago, il.

Cleckley, H. (1941). The mask of sanity; an attempt to reinterpret the so-called psychopathic personality. Mosby.

Cox, J. C., Friedman, D., \& Gjerstad, S. (2007). A tractable model of reciprocity 
and fairness. Games and Economic Behavior, 59, 17-45.

Curry, O., Chesters, M. J., \& Viding, E. (2011). The psychopath's dilemma: The effects of psychopathic personality traits in one-shot games. Personality and Individual Differences, 50(6), 804-809.

Declerck, C. H., Boone, C., \& Emonds, G. (2013). When do people cooperate? the neuroeconomics of prosocial decision making. Brain and cognition, 81(1), 95-117.

Deutsch, F. M. (1990). Status, sex, and smiling: The effect of role on smiling in men and women. Personality and Social Psychology Bulletin, 16(3), 531-540.

Ferrari, A., \& Comelli, M. (2016). A comparison of methods for the analysis of binomial proportion data in behavioral research. arXiv preprint arXiv:1605.01592, $1-15$.

Hess, U., Blairy, S., \& Kleck, R. E. (2000). The influence of facial emotion displays, gender, and ethnicity on judgments of dominance and affiliation. J. Nonverbal Behav., 24, 265-283.

Ibáñez, M. I., Sabater-Grande, G., Barreda-Tarrazona, I., Mezquita, L., LópezOvejero, S., Villa, H., ... Georgantzís, N. (2016). Take the money and run: psychopathic behavior in the trust game. Frontiers in psychology, 7, 1866.

Kätsyri, J., Hari, R., Ravaja, N., \& Nummenmaa, L. (2013). The opponent matters: elevated fmri reward responses to winning against a human versus a computer opponent during interactive video game playing. Cerebral Cortex, 23, 28292839.

Koenigs, M., Kruepke, M., \& Newman, J. P. (2010). Economic decision-making in psychopathy: a comparison with ventromedial prefrontal lesion patients. Neuropsychologia, 48(7), 2198-2204.

Kroenke, K., Spitzer, R. L., \& Williams, J. B. (2001). The phq-9: validity of a brief depression severity measure. Journal of general internal medicine, 16(9), 606- 
613.

Loewenstein, G. (2000). Emotions in economic theory and economic behavior. The American Economic Review, 90, 426-432.

Maes, J. H., Woyke, I. C., \& Brazil, I. A. (2018). Psychopathy-related traits and decision-making under risk and ambiguity: An exploratory study. Personality and Individual Differences, 122, 190-194.

Mao, A., Dworkin, L., Suri, S., \& Watts, D. J. (2017). Resilient cooperators stabilize long-run cooperation in the finitely repeated prisoner's dilemma. Nature communications, 8,13800 .

Mavadati, S. M., Mahoor, M. H., Bartlett, K., Trinh, P., \& Cohn, J. F. (2013). Disfa: A spontaneous facial action intensity database. IEEE Transactions on Affective Computing, 4, 151-160.

Mokros, A., Menner, B., Eisenbarth, H., Alpers, G. W., Lange, K. W., \& Osterheider, M. (2008). Diminished cooperativeness of psychopaths in a prisoner's dilemma game yields higher rewards. Journal of abnormal psychology, 117(2), 406.

Ohtsuki, H., Iwasa, Y., \& Nowak, M. A. (2015). Reputation effects in public and private interactions. PLoS Computational Biology, 11 .

Patrick, C., Fowles, D. C., \& Krueger, R. F. (2009). Triarchic conceptualization of psychopathy: Developmental origins of disinhibition, boldness, and meanness. Development and psychopathology, 21, 913-938.

Patrick, C. J. (2010). Operationalizing the triarchic conceptualization of psychopathy: Preliminary description of brief scales for assessment of boldness, meanness, and disinhibition. Unpublished test manual, Florida State University, Tallahassee, $F L$.

Rilling, J. K., Glenn, A. L., Jairam, M. R., Pagnoni, G., Goldsmith, D. R., Elfenbein, H. A., \& Lilienfeld, S. O. (2007). Neural correlates of social cooperation and 
non-cooperation as a function of psychopathy. Biological psychiatry, 61(11), $1260-1271$.

Scodel, A., Minas, J. S., Ratoosh, P., \& Lipetz, M. (1959). Some descriptive aspects of two-person non-zero-sum games. Journal of Conflict Resolution, 3(2), 114119.

van Rijsoort, S., Emmelkamp, P., \& Vervaeke, G. (1999). The penn state worry questionnaire and the worry domains questionnaire: Structure, reliability and validity. Clinical Psychology \& Psychotherapy: An International Journal of Theory \& Practice, 6(4), 297-307.

Widom, C. S. (1976). Interpersonal conflict and cooperation in psychopaths. Journal of Abnormal Psychology, 85, 330. 
Tables

Table 1. Payoff structure of each move in a typical Prisoner's

Dilemma game.

\begin{tabular}{lll} 
& Cooperate & Defect \\
\cline { 2 - 3 } Cooperate & $\mathrm{R}=3, \mathrm{R}=3$ & $\mathrm{~S}=0, \mathrm{~T}=5$ \\
\cline { 2 - 2 } Defect & $\mathrm{T}=5, \mathrm{~S}=0$ & $\mathrm{P}=1, \mathrm{P}=1$
\end{tabular}

$\mathrm{T}>\mathrm{R}>\mathrm{P}>\mathrm{S}, \mathrm{R}>(\mathrm{S}+\mathrm{T}) / 2$

Table 2. Payoff matrix showing the percentage profit earned during the game by each participant according to both players' decisions.

\begin{tabular}{cccc} 
& & \multicolumn{2}{c}{ Participant } \\
\cline { 3 - 4 } & & Standard Price & Sale Price \\
\hline \multirow{2}{*}{ Opponent } & Standard Price & $(30 \%, 30 \%)$ & $(10 \%, 40 \%)$ \\
\cline { 2 - 4 } & Sale Price & $(40 \%, 10 \%)$ & $(20 \%, 20 \%)$ \\
\hline
\end{tabular}


Table 3. Descriptive statistics for the participant sample

\begin{tabular}{|c|c|c|c|c|c|}
\hline Variables & Min & Mean & Standard Deviation & Max & Cronbach's $\alpha$ \\
\hline age & 18 & 27.26 & 8.92 & 65 & - \\
\hline gender & 1 & 1.43 & 0.50 & 2 & - \\
\hline ethnicity & 1 & 1.02 & 0.14 & 2 & - \\
\hline nationality & 1 & 1.05 & 0.22 & 2 & - \\
\hline education & 2 & 4.69 & 0.70 & 5 & - \\
\hline job & 1 & 3.57 & 1.47 & 6 & - \\
\hline degree & 1 & 2.83 & 1.74 & 8 & - \\
\hline believe & 1 & 2.06 & 1.31 & 5 & - \\
\hline maximise & 1 & 3.73 & 1.20 & 5 & - \\
\hline meanness & 19 & 30.99 & 7.95 & 67 & 0.87 \\
\hline boldness & 30 & 50.01 & 7.50 & 72 & 0.81 \\
\hline disinhibition & 21 & 33.58 & 7.93 & 62 & 0.87 \\
\hline
\end{tabular}

Gender: $1=$ Female, $2=$ Male

Ethnicity: 1 = European, 2 = other;

Nationality: 1 = German, 2 = other;

Education: 1 = Primary school, 2 = Secondary school, 3 = Gymnasium, 4 = High school, 5 = Higher school

Degree: $1=$ Still in education, $2=$ No education completed, $3=$ Apprenticeship, $4=$ Bachelor, $5=$ Master, $6=$ Doctoral

student, 7 = Doctor, $8=$ other

Job sector: $1=$ Technical, $2=$ Research, $3=$ Art and music, $4=$ Social, $5=$ Economic, $6=$ Administrative 


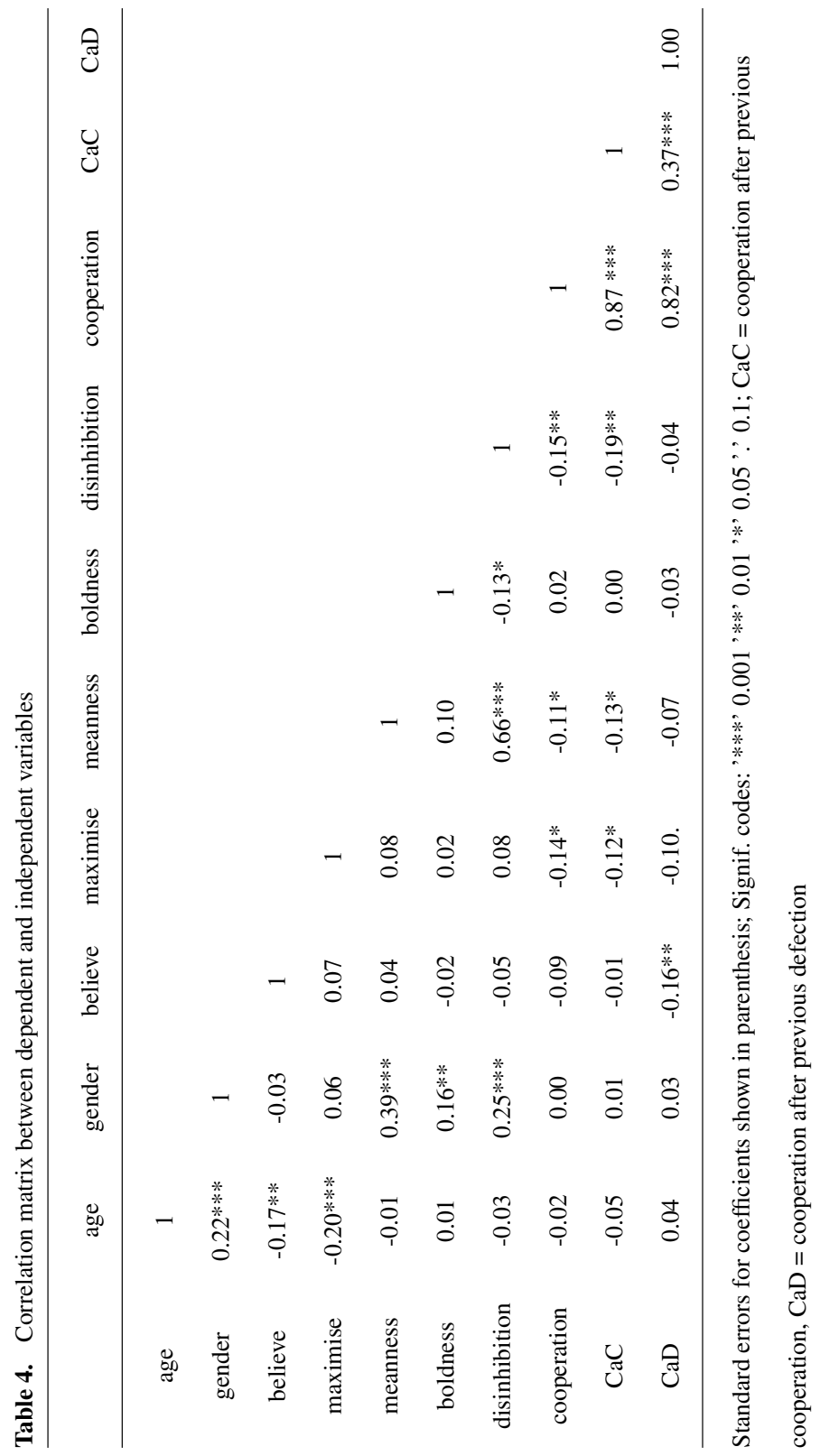


Table 5. GLM results for participants' cooperation as a function of their age, gender, level of belief and personality traits.

\begin{tabular}{|c|c|c|c|}
\hline DV: & overall cooperation & $\mathrm{CaC}$ & $\mathrm{CaD}$ \\
\hline \multirow[t]{2}{*}{ intercept } & $0.69 * * *$ & $0.58 * * *$ & $0.66 * * *$ \\
\hline & $(0.10)$ & $(0.12)$ & $(0.11)$ \\
\hline \multirow[t]{2}{*}{ age } & -0.003 & -0.00 & 0.00 \\
\hline & $(0.02)$ & $(0.00)$ & $(0.00)$ \\
\hline \multirow[t]{2}{*}{ gender } & 0.04 & 0.07 & 0.03 \\
\hline & $(0.04)$ & $(0.04)$ & $(0.01)$ \\
\hline \multirow[t]{2}{*}{ believe } & -0.02 & -0.01 & $-0.03^{*}$ \\
\hline & $(0.01)$ & $(0.01)$ & $(0.01)$ \\
\hline \multirow[t]{2}{*}{ maximise } & $-0.03 *$ & -0.04 & -0.02 \\
\hline & $(0.01)$ & $(0.02)$ & $(0.01)$ \\
\hline \multirow[t]{2}{*}{ meanness } & -0.08 & -0.01 & -0.02 \\
\hline & $(0.02)$ & $(0.03)$ & $(0.02)$ \\
\hline \multirow[t]{2}{*}{ boldness } & -0.02 & 0.01 & -0.01 \\
\hline & $(0.02)$ & $(0.02)$ & $(0.02)$ \\
\hline \multirow[t]{2}{*}{ disinhibition } & -0.04 . & $-0.06 *$ & 0.00 \\
\hline & $(0.02)$ & $(0.03)$ & $(0.02)$ \\
\hline
\end{tabular}

Standard errors for coefficients shown in parenthesis; Signif. codes: '***' $0.001{ }^{\prime} * *{ }^{\prime} 0.01{ }^{\prime}{ }^{\prime}$, 0.05 '. $0.1 ; \mathrm{CaC}=$ cooperation after previous cooperation, $\mathrm{CaD}=$ cooperation after previous defection 


\section{Appendix A. Binomial Regression analysis}

An important observation that arose from this analysis is how a model, which sometimes seems the perfect choice, can be in reality extremely far from a good representation of the dataset. A clear example for our dataset is the binomial regression. We expected this model to give the best interpretation of the dataset, as our dependent variable is a binary choice repeated over rounds, and so a binomial distribution might be expected to be the optimal choice to describe our dataset (Ferrari \& Comelli, 2016). See supplementary material for more extensive discussion. Nevertheless, we proved that such a model was the worst performing one out of the four analysed. Additionally, it is worth reporting that if we had used the binomial regression model, several of the predictor variables would have appeared to have a statistically significant effect on the level of cooperation as shown in Table A1. Looking at these results, it might have been tempting to select the binomial regression model as the preferred regression and not take into account the analysis implemented for model selection. However, our results highlight the importance of a correct model selection procedure for data analysis and show how easy it is to misinterpret the results of a regression. 
Table A1. Binomial regression results for participants' cooperation as a function of their age, gender, level of belief and personality traits.

\begin{tabular}{|c|c|}
\hline DV: & Overall Cooperation \\
\hline \multirow[t]{2}{*}{ intercept } & $0.82 * * *$ \\
\hline & $(0.19)$ \\
\hline \multirow[t]{2}{*}{ age } & $-0.01 * *$ \\
\hline & $(0.00)$ \\
\hline \multirow[t]{2}{*}{ gender } & $0.18 *$ \\
\hline & $(0.07)$ \\
\hline \multirow[t]{2}{*}{ believe } & $-0.09 * * *$ \\
\hline & $(0.02)$ \\
\hline \multirow[t]{2}{*}{ maximise } & $-0.14 * * *$ \\
\hline & $(0.03)$ \\
\hline \multirow[t]{2}{*}{ meanness } & -0.04 \\
\hline & $(0.04)$ \\
\hline \multirow[t]{2}{*}{ boldness } & -0.01 \\
\hline & $(0.03)$ \\
\hline \multirow[t]{2}{*}{ disinhibition } & $-0.18 * * *$ \\
\hline & $(0.04)$ \\
\hline
\end{tabular}

Standard errors for coefficients shown in parenthesis; Signif. codes: ${ }^{* * *}{ }^{\prime} 0.0011^{\prime} *{ }^{*}, 0.01{ }^{\prime}{ }^{*}, 0.05,, 0.1 ; \mathrm{BIN}=$ binomial generalised linear model 